\title{
Importancia de las teorías acerca de los colaboradores \\ en la gestión y liderazgo eficaces
}

FECHA DE RECEPCIÓN: 9 de agosto FECHA DE APROBACIÓN: 26 de octubre Pp.96-115

\section{Rodrigo Arturo Zárate T.*} Julio César Acosta P.**

\section{The importance of theories about employees in efficient leadership and} management

\section{Impartance des thécries sur} les collaborateurs pour une gestion et un leadership efficace

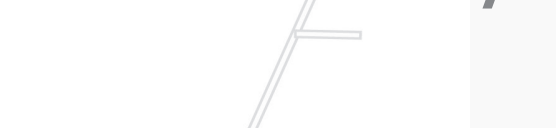

Importância das teorias sabre us colaboradores na gestã̃ e a liderança eficazes
* Doctor of Strategic Leadership-DSL, enfoque en Gerencia, Escuela de Liderazgo Global y Emprendimiento Regent University, Virginia Beach, USA. Certificate in Advance Graduate Studies Estudios a nivel de Maestría en Liderazgo Regent University, Virginia Beach, USA. Master of Business Administration - MBA, enfoque en Mercadeo y Negocios Internacionales, Regent University, Virginia Beach, USA. Ingeniero Industrial Universidad de América, Bogotá. Par evaluador certificado como conocedor de las normas de acreditación y evaluador de Instituciones Educativas que desean obtener acreditación internacional por la Accreditation Council for Business Schools and Programs ACBSP.

**Doctor en Dirección y Organización de Empresas de la Universidad Autónoma de Madrid. Docente Investigador y Coordinador de Investigación y Desarrollo de la Facultad de Admiitración de Empresas de la Universidad Externado de Colombia. 


\section{RESUMEN}

En la gran mayoría de las teorías de liderazgo se habla siempre de dos actores, los líderes y los colaboradores (también denominados, seguidores), sin embargo a estos últimos se les ha prestado poca atención. Son pocas las teorías acerca de los colaboradores que presenta la literatura actual. La presente investigación muestra una revisión de la literatura sobre los atributos de los colaboradores basado en el arcoíris de los atributos de los seguidores desarrollado por Antelo, Prilipko y Sheridan-Pereira (2010) y sus implicaciones en la gestión, que se traduce en la identificación de los atributos inhibidores y facilitadores de los colaboradores que coexisten en el entorno laboral. La identificación de estos atributos favorecerá la gestión del líder y, a su vez, la generación de condiciones de trabajo que facilitará tanto el flujo de información y conocimiento de los colaboradores como la disposición a establecer relaciones interpersonales eficaces orientadas al logro y obtención de resultados superiores en la organización.

\section{ABSTRACT}

In almost all leadership theories, two actors are always mentioned, leaders and collaborators (also known as followers); however, the latter ones haven't been the object of research. Today, very few works can be found on collaborators' theories. This research shows a bibliographic revision on collaborators' attributes based on the studies made by Antelo, Prilipko and Sheridan-Pereira (2010) and its implications on management, which is translated into the identification of inhibition factors and facilitators of collaborators interacting in a working environment. The identification of these attributes will favour leadership management and the generation of working conditions that facilitate either information flow and knowledge of collaborators or the disposition to establish goal-oriented interpersonal relationships and the procurement of better results with organizations.

\section{RESUMÉÉ}

Dans la grande majorité des théories du leadership il est fait référence aux deux acteurs-clé que sont les dirigeants et collaborateurs (également appelés disciples). Cependant, peu d'attention a été consacrée à ces derniers. Il n'existe en effet que peu de théories dans la littérature actuelle faisant référence aux collaborateurs. Cette investigation passe en revue la littérature existante sur les attributs des collaborateurs basés sur la panoplie des attributs des disciples développée par Antelo, Prilipko et Sheridan-Pereira (2010) et leurs implications sur la gestion. Cela se traduit par l'identification des attributs inhibiteurs et facilitateurs des collaborateurs qui coexistent dans le monde professionnel. L'identification de ces attributs améliorera la gestion du leader mais également la création de conditions de travail qui facilitera la circulation de l'information et des connaissances des collaborateurs comme la volonté d'établir des

\section{RESUMO}

Na grande maioria das teorias de liderança fala-se sempre de dois atores, os líderes e os colaboradores (também chamados, seguidores); contudo, os seguidores tem recebido pouca atenção. A literatura atual apresenta poucas teorias sobre os colaboradores. Esta pesquisa mostra uma revisão da literatura sobre os atributos dos colaboradores baseado no arco-íris dos atributos dos seguidores desenvolvido por Antelo, Prilipko e Sheridan-Pereira (2010) e suas implicações na gestão, que leva a identificar os atributos inibidores e facilitadores dos colaboradores que coexistem no entorno de trabalho. A identificação destes atributos favorecerá a gestão do líder e, ao mesmo tempo, a geração de condições de trabalho que facilitarão o fluxo de informação e conhecimento dos colaboradores e a disposição para estabelecer relações interpessoais eficazes orientadas a atingir e obter resultados superiores na organização.

\section{Palabras claves}

Seguidores

Atributos de los colaboradores

Liderazgo

Gestión

\section{Key words}

Followers

Collaborators' attributes

Leadership

Management

\section{Mots clefs}

Disciples

Attributs des collaborateurs

Leadership

Gestion

\section{Palavras-chave}

\author{
Seguidores \\ Atributos dos colaboradores \\ Liderança \\ Gestão
}




\section{INTRODUCción}

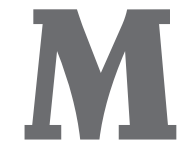

ientras la literatura acerca del liderazgo abunda, existe poca literatura acerca de los colaboradores (Thornborrow, 1994; Brown y Thornborrow, 1996; Bennis, 2008). En esta era de alta tecnología donde los colaboradores están cambiando, los líderes y gerentes tienen que cambiar también (Brown, 2003), como Kellerman (2008) menciona, la tecnología hace difuso el poder y por lo tanto los líderes deben cambiar conociendo como son y como están cambiando sus colaboradores.

Adicionalmente, ni el liderazgo ni la gerencia podrían existir sin colaboradores (Hollander y Kelley, 1992; Lundin y Lancaster, 1990; Vecchio, 1987), sin embargo, se ha prestado mayor atención a las personas que ocupan altos cargos donde demuestran prestigio y poder, especialmente en culturas donde el líder es visto como una figura heroica (Calder, 1977; Konst, Vonk y Van der Vlist, 1999; Meindl, Ehrlich, y Dukerich, 1985; Pfeffer, 1977; Yukl, 2002).

Existen muchos factores que determinan como los colaboradores contribuyen en la efectividad de los líderes y los gerentes (Awamleh y Gardner, 1999; Choi y Mai-Dalton, 1999; Lord y Maher, 1991; Meindl et al, 1985; Yukl, 2002). Uno de esos factores es que la medición que se realiza al equipo o la organización del líder, es decir, un líder o gerente es catalogado más efectivo conforme al desempeño y éxito de su grupo o equipo de colaboradores.

Existen dos razones principales por las cuales se debe estudiar a los colaboradores (Buhler, 1993; Brown y Thornborrow, 1996), la primera es porque se puede llegar a un mayor entendimiento del liderazgo y la segunda, porque se puede llegar a un mayor entendimiento de los colaboradores y así crear procesos de capacitación para lograr el éxito de las organizaciones.

Las teorías existentes hablan de la efectividad del seguidory algunas de ellas presentan guías para lograr tal efectividad (Chaleff, 1995; Kelley, 1992; Whetton y Cameron, 1991). Con base a estos argumentos, realizamos una revisión de las teorías acerca de los colaboradores para conocer su importancia en el liderazgo y gestión de las organizaciones.

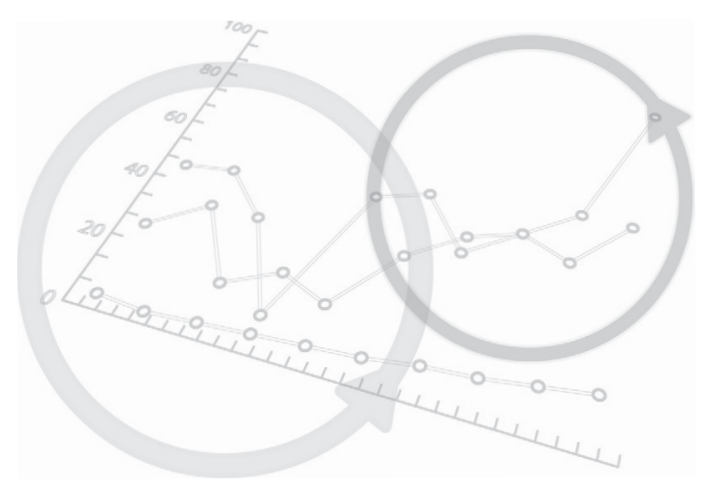




\section{LA DINÁMICA DE LOS COLABORADORES}

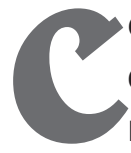

omo uno de los precursores en el tema de los colaboradores o seguidores, Abraham Zaleznik (1965) escribe acerca de la dinámica de los colaboradores clasificando los patrones y las dinámicas de los mismos en cuatro grandes grupos influenciados por el control y el comportamiento.
Estos cuatro grupos en los cuales se pueden clasificar a los colaboradores son: impulsivos, compulsivos, masoquistas y retraídos, los cuales se explicaran más adelante.

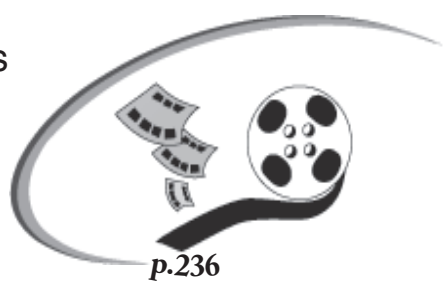

Figura 1. Clasificación de los colaboradores según Zaleznik

\begin{tabular}{|c|c|c|c|}
\hline \multicolumn{4}{|c|}{$\begin{array}{c}\text { DOMINANCIA } \\
\text { (Deseo de controlar) }\end{array}$} \\
\hline $\begin{array}{c}\text { Comportamiento } \\
\text { ACTIVO }\end{array}$ & Impulsivo & Compulsivo & $\begin{array}{c}\text { Comportamiento } \\
\text { PASIVO }\end{array}$ \\
\hline & $\begin{array}{r}\text { Masoquista } \\
\text { SUn } \\
\text { (Deseo de s }\end{array}$ & $\begin{array}{l}\text { Retraído } \\
\text { rolado) }\end{array}$ & \\
\hline
\end{tabular}

Fuente. Adaptado de Dynamics of Subordinacy (Zaleznik, 1965)

Zaleznik (1965) (Figura 1), menciona que en los colaboradores el llevar los conflictos de lo interno a expresarlos en su propio comportamiento, se da en respuesta a la relación vertical, es decir con su líder (Zaleznik, 1965). También menciona, que esta relación vertical se refleja y es reflejada en la constelación de los motivos, deseos y tensiones de cada individuo, y que se pueden determinar dos tendencias en esta constelación (Zaleznik, 1965). La teoría de Zaleznik se basa en conflictos que se presentan en la relación líder-colaborador.
La primera de estas tendencias tiene que ver con la dominancia y la sumisión. Zaleznik, (1965) menciona que la fuente potencial de conflicto aquí es por un lado el deseo de controlar y tener poder sobre las figuras de autoridad y por el otro lado, el deseo de ser controlado y dominado por las mismas figuras de autoridad. Zaleznik (1965) menciona además que estos dos extremos llevan a un mismo fin, asegurar la aceptación de las figuras de autoridad que regulan las recompensas y los castigos, y que a nivel organizacional, la intensidad de las reacciones de los colaboradores está generalmente relacionado con las oportunidades de asensos y éxito en la vida profesional. 
La segunda tendencia tiene que ver con el comportamiento de los colaboradores, donde este comportamiento puede ser activo o pasivo. Zaleznik (1965) menciona que en el extremo activo, los colaboradores toman la iniciativa y se involucran en sus propios entornos, mientras que en el extremo pasivo, los colaboradores esperan a que otros tomen la iniciativa y responden a los estímulos que vienen de fuera de ellos. Estos dos extremos tienen especial significado en la economía personal de cada individuo, pues reflejan las tensiones de recompensa y frustración, de energía involucrada y recompensa obtenida, de los riesgos que provienen de la frustración y de la necesidad de protegerse de dichos riesgos.

De las dos tendencias mencionadas, Zaleznik (1965) deriva las cuatro clasificaciones de colaboradores. Estas cuatro clasificaciones o tipos, reflejan el conflicto interno de los subordinados con sus líderes:

\subsection{Colaborador impulsivo}

De acuerdo con Zaleznik (1965), la característica principal de un colaborador que intenta dominar su relación con la figura de autoridad a través de la actividad es la rebelión, sin embargo, cuando el colaborador impulsivo y rebelde logra controlar sus propias fantasías, un comportamiento constructivo aparece y el colaborador puede convertirse en espontáneo y valiente. Estas dos últimas características moldean al colaborador y puede utilizar su rebeldía para hablar francamente y presentar sus puntos de vista ante los problemas en el trabajo, evita la conformidad, no solamente por la impaciencia, sino por la urgencia de crear y lograr. El uso constructivo de la dominancia y la actividad puede lograr en los colaboradores que tengan influencia en eventos al interior de las organizaciones.

La línea de separación entre el comportamiento constructivo y destructivo es muy delgada, la diferencia radica en el autocontrol del individuo. El rebelde constructivo sabe cómo usar su deseo de dominar y actúa de forma apropiada, mientras que el rebelde destructivo no tiene control de sí mismo y se deja llevar por sus propias emociones y fantasías (Zaleznik, 1965).

\subsection{Colaborador Compulsivo}

Zaleznik (1965) define al colaborador compulsivo como aquel que desea dominancia y control a través de un comportamiento pasivo. La diferencia entre compulsivo e impulsivo, aunque es confusa, radica en que un individuo impulsivo actúa sin pensar, mientras que un individuo compulsivo piensa de manera excesiva antes de actuar demostrando así una conciencia poderosa y fuertes sentimientos de culpabilidad.

Zaleznik (1965) menciona cuatro cualidades recurrentes de los colaboradores compulsivos, duda, ambigüedad, agresión oculta y negación de responsabilidad. La duda en los colaboradores compulsivos está presente generalmente en el desarrollo de su carrera profesional, es decir en las decisiones que debe tomar y la profesión o cargos que debe perseverar. La ambigüedad se presenta especialmente en la contradicción de sus propias emociones hacia objetos o eventos específicos. Esta cualidad va de la mano con la duda y entre las dos crean tensión en las relaciones del colaborador y su actividad en el trabajo.

La tercera de las cualidades que menciona Zaleznik (1965) es la agresión oculta, donde el colaborador genera intencionalmente argumentos que provocan conflicto. Los motivos del colaborador provienen de un desbalance entre sus propios deseos de dependencia e independencia. La cuarta de las cualidades, es la negación de responsabilidad, la cual se refiere a que los conflictos, problemas y las emociones negativas provienen de fuera del individuo y no de su interior. 


\subsection{Colaborador masoquista}

Zaleznik (1965) menciona que el masoquismo consiste en sentir placer en el dolor y que el verdadero masoquismo es un problema emocional. En cuanto a los colaboradores, aquel que es masoquista es el que desea el dolor sometiéndose al control y asertividad de la autoridad. Zaleznik (1965) aclara que en este caso, el masoquismo es un aspecto de motivación inconsciente. Esta motivación es generalmente de autodestrucción y lo logra a través de provocar a otros.

Una de las manifestaciones más comunes de los colaboradores masoquistas, es aquel que se encuentra siempre involucrado en accidentes (de todo tipo) en la organización, es quien siempre se encuentra molesto con su jefe (figura de autoridad) pero solamente logra herirse asimismo logrando lapsos de atención o tomando riesgos innecesarios.

\subsection{Colaborador retraído}

El cuarto grupo o tipo de colaboradores, son los retraídos. Zaleznik (1965) menciona que estos representan un resultado de lograr trasladar la atención del mundo exterior hacía ellos mismos. Este comportamiento proviene de la falta de confianza, aquí el mundo se ve malévolo y por lo tanto el individuo se tiene que retraer de él. El colaborador retraído presenta una seria dificultad en su relación con sus superiores, presenta falta de confianza, interés e involucramiento que lo hace difícil de influenciar, hace lo que se le pide pero sin ningún interés, contribuye poco al cambio y es poco innovador, puede manejar muy bien la rutina, pero hace muy poco más allá de las demandas necesarias de su trabajo; entre otras características del colaborador retraído, este parece leal y además parece que aceptara los estándares existentes (Zaleznik, 1965).

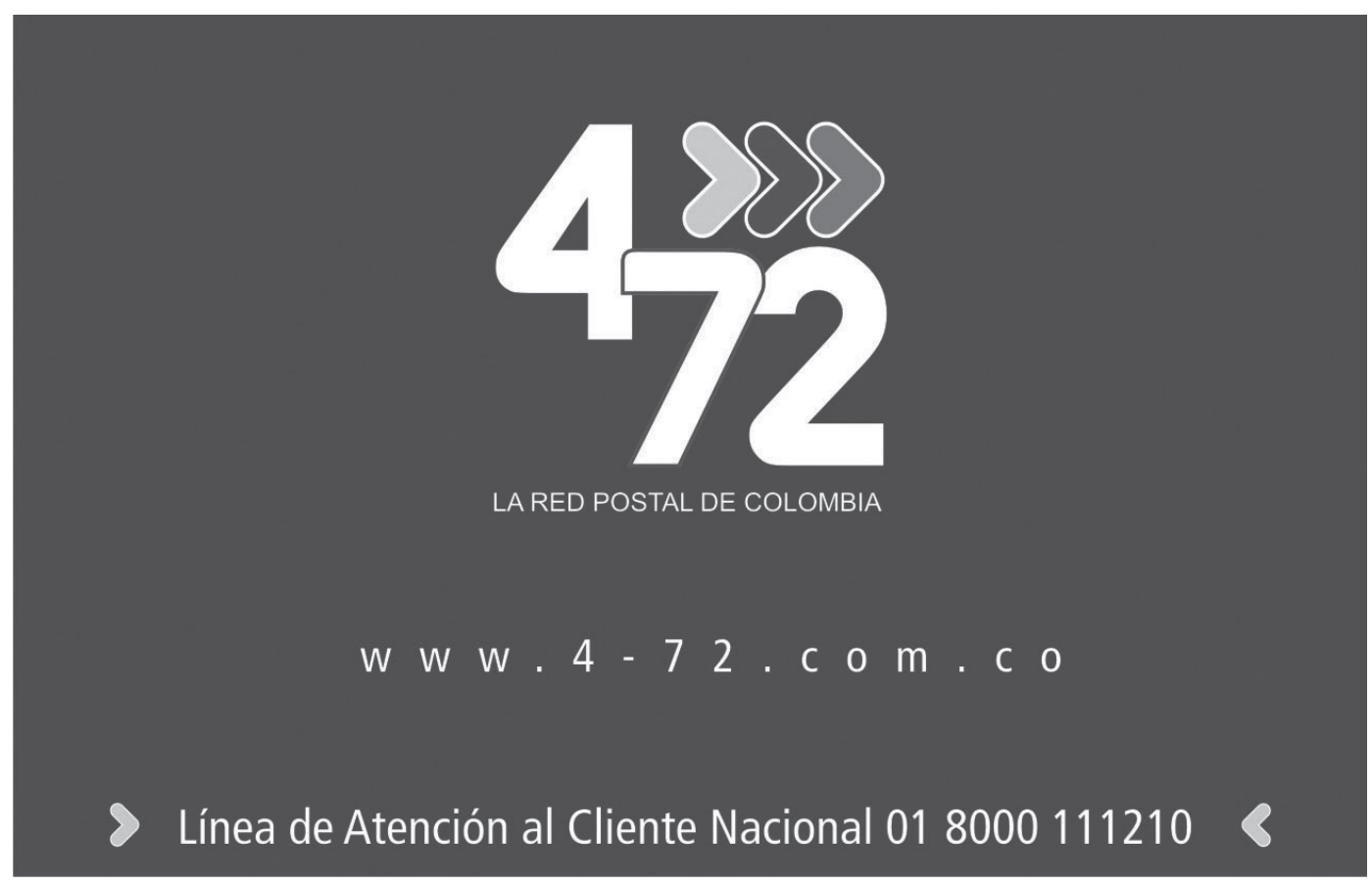




\section{ESTILOS DE COLABORADORES}

E n términos académicos, el segundo autor en referirse a los colaboradores fue. Kelley (1992), quien describe cinco estilos de colaboradores basado en dos dimensiones las cuales definen la manera en que ellos actúan. La primera dimensión se refiere a la manera en que piensan, en si son independientes o dependientes en la forma en que piensan y la segunda dimensión se refiere a: si son pasivos o activos a la hora de involucrarse en los asuntos de la organización. De estas dos dimensiones surgen cinco estilos de colaboradores de acuerdo con Kelley (1992),

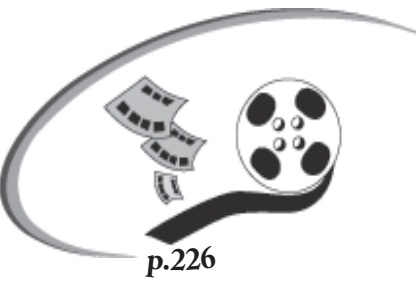
los pasivos, los conformistas, los alineados, los pragmáticos y los efectivos (figura 2).

Figura 2. Estilos de colaboradores según Kelley

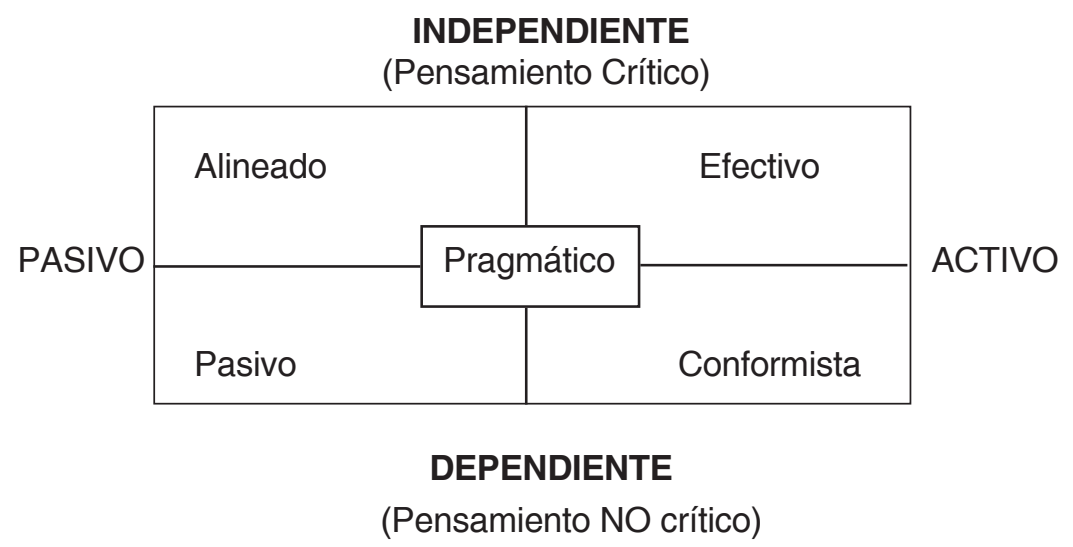

Fuente. Adaptado de The Power of Followership (Kelley, 1992)

Cada una de las dimensiones son la base para cada uno de los estilos señalados. Las dimensiones de acuerdo con Kelley (2008) responden a diferentes preguntas, la dimensión de dependencia-independencia responde a las preguntas que se hacen a los colaboradores tales como: ¿Piensan por ellos mismos? ¿son pensadores críticos independientes o buscan que su líder piense por ellos? Así mismo, la dimensión activo-pasivo responde a preguntas tales como: ¿Están comprometidos en crear una energía positiva para la organización o existe energía negativa o involucramiento pasivo?

\subsection{Estilo pasivo}

El estilo pasivo es también llamado la oveja por Kelley (2008). La comparación se hace porque las ovejas son pasivas y buscan al líder para que piense por ellas y las motive. Kelly (2008) como un ejemplo menciona que si usted va en su auto y piensa que va a poner a hacer a sus empleados y como va a lograr que ellos lo hagan, entonces usted tiene ovejas a su cargo.

El colaborador de este estilo no exhibe un pensamiento crítico ni una participación activa en la organización. Este colaborador no tiene iniciativa ni 
sentido de responsabilidad, se deja llevar por su líder. Generalmente los colaboradores pasivos son el resultado de líderes supremamente controladores, autoritarios y coercitivos (Kelley, 1992).

\subsection{Estilo conformista}

El colaborador conformista es también llamado por Kelley (2008) el colaborador que 'dice si a todo'. Los colaboradores conformistas son positivos, siempre están del lado del líder y siempre buscan de su líder que piense por ellos, que les de dirección y visión, estos colaboradores siempre tienen energía para hacer lo que su líder les pide que hagan y una vez lo hacen, vuelven a su líder preguntando que más pueden hacer (Kelley, 2008).

El colaborador conformista es activo pero dependiente, participa activamente sin pensar o tener en cuenta las consecuencias, dice si a todo lo que le pide su líder.

\subsection{Estilo alienado}

De acuerdo a Kelley (2008) el colaborador alienado piensa por sí solo pero tiene demasiada energía negativa, cada vez que la organización intenta moverse hacia adelante, este estilo de colaborador tiene bastantes razones de porque ni la organización ni el líder deberían hacerlo. Kelley (2008) también menciona que estos colaboradores son inteligentes, tienen energía pero no la utilizan positivamente para ir hacia la dirección correcta, que ellos creen que son los únicos en la oficina que son capaces de enfrentar a sus jefes.

\subsection{Estilo pragmático}

El colaborador pragmático es que de acuerdo a Kelley (2008) siempre está viendo como están las cosas y se ajusta a su entorno y una vez determinan hacia donde se dirige su entorno ajustan su comportamiento y se dirigen hacía donde se dirige el entorno. Kelley (2008) además menciona que los colaboradores pragmáticos nunca serán los primeros en acompañar una decisión, pero siempre estarán acompañando las decisiones que tome la organización o sus líderes.

El colaborador pragmático tiene todas las dimensiones y se puede ajustar a cualquier situación por la que esté pasando la organización, él solamente espera a ver cuál es la situación y ajusta su comportamiento a dicha situación.

\subsection{Estilo efectivo}

Los colaboradores efectivos son también llamados por Kelley (2008) los colaboradores estrella, pues piensan por sí mismos, tienen energía positiva y son activos. Ellos evalúan todas las decisiones de su líder y si acuerdan con el líder, le dan todo su apoyo y si no acuerdan con el líder, entonces desafían al líder siempre de forma apropiada y ofreciendo alternativas de solución.

Los colaboradores efectivos tratan a todo el mundo por igual sin importar su cargo, además que nunca intentan evadir ni el riesgo ni el conflicto, son activos y piensan críticamente. 


\section{EL COLABORADOR AUDAZ}

La cultura occidental define al colaborador en términos

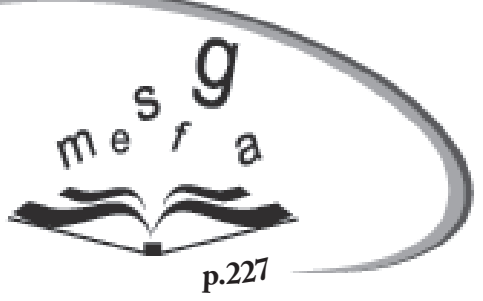

de conformidad, pasividad y debilidad y lo ubica en un nivel inferior al líder (Chaleff, 1995; Chaleff, 1997; Chaleff, 2010; Yukl, 2002). De acuerdo a Yukl (2002) esta connotación es influenciada por las experiencias vividas tanto en casa como en el colegio, donde otros eran responsables por el comportamiento del individuo, pero este no era responsable por el comportamiento de los otros.

Figura 3. Tipología de los colaboradores según Chaleff
Chaleff (1995) escribe de la importancia de los seguidores y menciona que tanto los líderes como los seguidores orbitan alrededor del propósito de la organización y que en ningún momento los seguidores orbitan alrededor de los líderes. Chaleff (1995) define que existen dos directrices que enmarcan la tipología de los colaboradores, una directriz que se refiere al grado de apoyo que le brinda el colaborador al líder y la otra directriz que se refiere al grado en el cual el colaborador desafía al líder. Del cruce de estas dos directrices, (figura 3), Chaleff (1995) determinó los cuatro tipos de seguidores que considera en su tipología, los socios, los implementadores, los recursos y los individualistas.

\begin{tabular}{|c|c|c|c|}
\hline \multicolumn{4}{|c|}{ APOYA A LOS LIIDERES } \\
\hline DESAFÍA LOS & Socios & Implementadores & \multirow{2}{*}{$\begin{array}{l}\text { NO DESAFÍA A } \\
\text { LOS LÍDERES }\end{array}$} \\
\hline & Individualistas & Recursos & \\
\hline & NO APOY & OS LIÍDERES & \\
\hline
\end{tabular}

Fuente. Adaptado de The Corageous Follower Chaleff, (1995)

Ser buen colaborador es visto como una habilidad que requiere valentía mientras que obedecer ciegamente no es necesariamente la cualidad de un buen colaborador, por otro lado ser buen líder significa ser buen colaborador y viceversa (Chaleff, 1995). La tipología determinada por Chaleff es la siguiente:

\subsection{Implementadores}

Los implementadores de acuerdo a Chaleff (1995) son aquellos colaboradores que toman una orden y la cumplen sin cuestionar absolutamente nada, es decir que apoyan totalmente al líder pero no lo desafían en absoluto. 


\subsection{Socios}

De acuerdo a Chaleff (1995), a los colaboradores socios les gusta ser tratados como iguales por los líderes, aunque respetan la posición del líder, son fuertes apoyadores, pero cuestionaran al líder cuando ellos lo consideren necesario.

\subsection{Individualistas}

Chaleff (1995) menciona de los individualistas que no son colaboradores fáciles de llevar, puesto que les gusta hacer las cosas a su manera desafiando al líder y sin brindarles apoyo.

\subsection{Recursos}

De este último tipo de seguidores Chaleff (1995) menciona que hacen lo que les es solicitado únicamente, es decir lo suficiente para no ser cuestionados ni en ninguna manera amonestados, son obedientes pero no tienen la inteligencia o valentía suficiente para desafiar al líder. 


\section{EL CONTINUUM DE BÁRBARA}

L as diferencias entre los seguidores son consecuencia directa de aquellas entre los líderes y tiene implicaciones críticas en la forma en que los gerentes deben gerenciar (Kellerman, 2007).

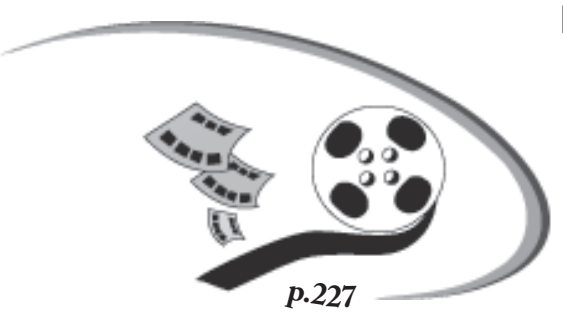

Kellerman categoriza a los colaboradores en un continuum (figura 4) que va de sentir y hacer absolutamente nada a estar apasionadamente comprometido y profundamente involucrado. Kellerman (2007) menciona que finalmente es el grado de compromiso que tenga el colaborador lo que determina su relación con el líder.

Figura 4. El continuum de Kellerman

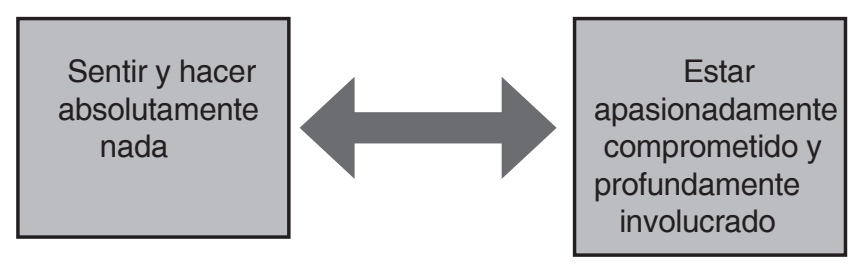

Fuente. Adaptado de What Every Leader Needs to Know about Followers (Kellerman, 2007)

Dentro del continuum de Kellerman, la escritora define cinco categorías de colaboradores, los aislados, los desentendidos, los participantes, los activistas y los comprometidos (Kellerman, 2007) (figura 5). La definición de cada una de las categorías se encuentra a continuación.
Figura 5. Categorización de los colaboradores según Kellerman

\begin{tabular}{|c|c|c|c|c|}
\hline \multicolumn{2}{|c|}{$\begin{array}{l}\text { Sentir y hacer } \\
\text { absolutamente } \\
\text { nada }\end{array}$} & \multicolumn{2}{|c|}{ CONTINUUN } & $\begin{array}{c}\text { Estar } \\
\text { apasionadamente } \\
\text { comprometido y } \\
\text { profundamente } \\
\text { involucrado }\end{array}$ \\
\hline Aislado & Desentendido & Participante & Activisa & Comprometido \\
\hline
\end{tabular}

Fuente. Adaptado de What Every Leader Needs to Know about Followers (Kellerman, 2007)

\subsection{Colaborador aislado}

Los colaboradores aislados están completamente desinformados de lo que sucede alrededor de ellos, no les importa lo que está sucediendo con sus líderes ni con la organización, al no hacer y saber nada, esta categoría de colaborador soporta el quehacer diario de la organización pasivamente (Kellerman, 2007).

Esta categoría de colaboradores se encuentra mayormente en grandes compañías, donde por el tamaño de la organización fácilmente pueden pasar desapercibidos (Kellerman, 2007). De acuerdo con Kellerman (2007), lo primero que tienen que hacer las organizaciones es identificar si tienen colaboradores aislados en su personal y luego tomas algún tipo de acción para lograr sacar de su aislamiento a estos colaboradores.

\subsection{Colaborador desentendido}

Son aquellos colaboradores que observan pero no participan (Kellerman, 2007). Este tipo de colaboradores hacen cuando es parte de sus propios intereses la actividad a realizar, pero no están internamente 
motivados a hacer y al igual que los aislados, los desentendidos pueden bajar el ánimo en los grupos en los cuales están involucrados (Kellerman, 2007).

\subsection{Colaborador participante}

A medida que se mueve el continuo, se va observando como cada categoría se va desplazando hacia el lado opuesto, y en este caso, los participantes ya se comprometen, tanto con su líder como con la organización, de alguna manera (Kellerman, 2007). Desde esta categoría en adelante los colaboradores toman acción dentro de la organización y la pregunta aquí es si lo hacen a favor o en contra de la organización.

\subsection{Colaborador activista}

De acuerdo a Kellerman (2007), los activistas tienen energía y están comprometidos, ellos invierten bastante tanto en las personas como en los procesos y están listos a apoyar al líder o por el contrario a desafiarlo y si es el caso lograr que el líder salga de la compañía. Muchos activistas son motivados por sus superiores a tomar acción y resolver situaciones bajo su propio criterio.

\subsection{Colaborador comprometido}

Estos colaboradores de acuerdo a Kellerman (2007) son totalmente devotos de sus líderes y están totalmente comprometidos tanto con su líder como con la organización, pero además puede ocurrir lo contrario, que se opongan firmemente a su líder por causa de la organización, es decir que los colaboradores comprometidos, pueden dirigir su devoción hacia una persona o hacia una causa (la organización). 


\section{ARCoÍRIS DE ATRIBUTOS DE LOS SEGUIDORES}

D e acuerdo con la definición propuesta en el Diccionario de la Lengua Española (2010), un atributo constituye "cada una de las cualidades o propiedades de una ser". Dicho esto, entendemos que un atributo es una cualidad, característica o propiedad que pertenece a la persona, cosa, grupo, etc.

La noción de atributo puede ser extendida por el concepto de la teoría de la atribución desarrollada por Kelley (1992), esta teoría señala que el liderazgo es una atribución que las personas asumen acerca de otros individuos. Usando el marco de la atribución, los investigadores (Stogdill, 1963, 1974; Kelley, 1973; Calder, 1977; Lord y Smith, 1983; Meindl, 1995; Waldman et al, 2001); han encontrado que las personas caracterizan a los líderes como dueños de atributos generales como inteligencia, personalidad desenvuelta, habilidades verbales, audacia, determinación, y comprensión, entre otros. A nivel organizacional, el marco de la atribución da razón de las condiciones bajo las cuales las personas usan el liderazgo para explicar los resultados organizacionales. Es decir, cuando una organización tiene un desempeño, ya sea negativo o positivo, las personas están dispuestas a establecer atribuciones del liderazgo para explicar el desempeño. Un tema muy relevante que comprende la teoría de la atribución del liderazgo, es la percepción de que los líderes eficaces son considerados en general, consistentes, y que no dudan cuando toman decisiones. Esto ayuda a explicar las relaciones causa-efecto, que se producen cuando un gerente es vulnerable en la organización debido a un revés financiero, a pesar de que no tuviera nada que ver con ello. También explica por qué estos gerentes tienden a obtener el crédito por los resultados financieros positivos, una vez más, a pesar de que no hayan contribuido a ello.

Dicho esto, el presente documento utiliza el instrumento desarrollado por Antelo, Prilipko y SheridanPereira (2010), que identifica y mide 12 atributos de los seguidores, los cuales son soportados por teorías, ejemplos y comparaciones y se explican a continuación.

\subsection{Facilidad para relaciones interpersonales}

Aunque la literatura ofrece poca información acerca de la gerencia de relaciones interpersonales (Clydesdale, 2009), este primer atributo hace referencia al desarrollo de relaciones con sus compañeros de trabajo, o relaciones interpersonales con otros colaboradores 0 seguidores, a partir del intercambio de emociones, afectos, necesidades, intereses y hasta formas de hacer y entender las cosas para el desarrollo de una tarea o actividad (Hunsaker y Cook, 1986).

La meta final de los colaboradores o seguidores efectivos es persistir en cultivar un clima de trabajo interpersonal con otros miembros del equipo que incluye respeto y relaciones armoniosas que se dirige a crear sentimientos de aceptación y de auto eficacia en el lugar de trabajo (Katz y Khan, 1960; Likert, 1968; Stodgill, 1974). 


\subsection{Facilidad para las relaciones y funciones en grupo}

De acuerdo a Menkes (2005), un gran pensamiento nunca comienza ni termina con una sola persona, gente talentosa trabajando junta pueden desempeñarse mucho mejor que si trabajaran solos. Este atributo hace referencia a las expectativas que se crea en las personas alrededor y con las cuales trabaja el colaborador. Antelo, Prilipko y Sheridan-Pereira (2010) mencionan que este atributo se fundamenta en la facilidad que tienen los colaboradores de disminuir conflictos con otros colaboradores (Marín y Troyano, 2006).

\subsection{Tolerancia}

Conforme a la definición del Diccionario de la Lengua Española (2010), tolerancia es el respeto a las ideas, creencias o prácticas de los demás cuando son diferentes o contrarias a las propias. Dicho esto, entendemos que es la capacidad de saber escuchar y aceptar a los demás, valorando las distintas formas de entender y posicionarse en la vida. La tolerancia si es entendida como respeto y consideración hacia la diferencia, como una disposición a admitir en los demás una manera de ser y de obrar distinta a la propia, o como una actitud de aceptación del legítimo pluralismo, es a todas luces un atributo de enorme importancia.

De acuerdo con Antelo, Prilipko y Sheridan-Pereira (2010), se espera de los colaboradores que exhiban una cantidad razonable de tolerancia trabajando solos o en un equipo, adicionalmente, de acuerdo a estos autores, en la práctica la tolerancia indica el apoyo de los colaboradores a las prácticas, acciones o decisiones que toman la gerencia y compañeros de trabajo a pesar de su desacuerdo en dichas acciones.

\subsection{Entendimiento conceptual}

Antelo, Prilipko y Sheridan-Pereira (2010), dicen que Zaccaro (2000) presentó la evidencia empírica acerca de la relación directa que existe entre un alto nivel de habilidad conceptual y el desempeño. Antelo, Prilipko y Sheridan-Pereira (2010) adicionan que esta habilidad es diferente al concepto de habilidad mental $\mathrm{o}$ inteligencia.

Este atributo hace referencia a la habilidad que expresan los seguidores a la hora de tomar decisiones puesto que para hacerlo de una manera rápida y eficaz, se requiere de un entendimiento conceptual de los temas a los que se refieren las decisiones o tareas a ejecutar.

\subsection{Facilidad de aprender y enfrentar el cambio}

Según Antelo, Prilipko y Sheridan-Pereira (2010), en un universo físico el cambio es una constante. Aún más cuando las organizaciones tienen la influencia tanto del entorno externo como del interno, el cambio es constante y requiere que los colaboradores estén prestos a asumir el cambio, enfrentarlo y aprender de dichos cambios (Senge, 1993).

Antelo, Prilipko y Sheridan-Pereira (2010), mencionan que un prerrequisito para aprender y enfrentar el cambio es el pensamiento crítico, ya que éste es razonable y reflexivo que se enfoca en que pensar o hacer y que requiere la habilidad de reconocer problemas, reunir e interpretar información, valorar las evidencias y evaluar líneas de pensamiento, puntos de vista, y opiniones personales que contribuyen a formar una acción basada en lo lógico, efectivo y real (Pierce, 1990). 


\subsection{Facilidad para la comunicación efectiva}

La habilidad para poder transmitir noticias positivas como negativas en la manera más apropiada requiere experiencia, coraje y habilidad (Antelo, Prilipko y Sheridan-Pereira 2010). El reto de los colaboradores es poder comunicar nuevas perspectivas con respeto a pesar de que esas ideas sean aceptadas o no (Pacheco, 2005). Este atributo hace referencia a la habilidad de los colaboradores de poder comunicar con eficiencia tanto no verbal como verbalmente.

\subsection{Consistencia como miembro de un grupo}

La consistencia como miembro de un grupo es clave en la reputación de los colaboradores en el lugar de trabajo (Antelo, Prilipko y Sheridan-Pereira, 2010). Excelentes colaboradores confían y trabajan efectivamente con otros, enfrentan el cambio y se desempeñan eficientemente, esto puede ocurrir cuando un colaborador es constante puesto que crea ese ambiente de apoyo mutuo (Antelo, Prilipko y Sheridan-Pereira, 2010; Latour y Rast, 2004). La consistencia crea confianza entre los miembros del grupo, lo cual incrementa las relaciones y por consiguiente el desempeño (Marín y Troyano, 2006).

\subsection{Facilidad para hacer contribuciones al grupo}

Los colaboradores tienen la responsabilidad de crear una cultura que evoque o haga florecer la creatividad y talentos internos de cada miembro del grupo y además que apoye el deseo natural de cada individuo de contribuir con el grupo (Marín y Troyano, 2006; Antelo, Prilipko y Sheridan-Pereira, 2010). Este atributo hace referencia no solamente a la contribución que hace el colaborador al grupo sino a su habilidad para ayudar a formar esa cultura donde todos los colaboradores deseen contribuir.

\subsection{Inteligencia emocional}

Este atributo es definido por Salovey y Mayer (1990), como la habilidad de percibir emociones, generar emociones para apoyar pensamientos, entender emociones y conocimiento emocional y regular las emociones para lograr un crecimiento intelectual y emocional. Dicho esto, la inteligencia emocional es un atributo que permite tomar conciencia de nuestras emociones, comprender los sentimientos de los demás, tolerar las presiones y frustraciones que soportamos en el trabajo, acentuar nuestra capacidad de trabajar en equipo y adoptar una actitud empática y social, que nos brindará mayores posibilidades de desarrollo persona y profesional (Goleman 1995, 2000).

Dentro de este atributo y de acuerdo a Antelo, Prilipko y Sheridan-Pereira (2010), es necesario definir el termino habilidad política, el cual hace referencia a la habilidad de entender a otros en el lugar de trabajo y usar ese conocimiento para influenciar a los demás para lograr objetivos tanto organizacionales como personales (Ferris et al, 2005).

Este atributo se refiere no solamente a la inteligencia emocional sino a la habilidad de usar dicha inteligencia para generar un mejor desempeño y así lograr avanzar hacia los objetivos organizacionales (Salovey y Sluyter, 1997).

\subsection{Facilidad de apoyar a otros}

La facilidad de apoyar a otros no hace referencia solamente a la habilidad de ofrecer apoyo emocional, sino además de ofrecer apoyo a las ideas innovadoras y creatividad de los compañeros de trabajo (Antelo, Prilipko y Sheridan-Pereira, 2010). De acuerdo a Antelo, Prilipko y Sheridan-Pereira (2010), este atributo también está asociado a la habilidad de los colaboradores de resolver situaciones problema (LEWIN, 1968). 


\subsection{Flexibilidad}

Antelo, Prilipko y Sheridan-Pereira (2010) mencionando la teoría de Kolb (1984) mencionan que la habilidad para responder flexiblemente al cambio tiene una fuerte influencia en la adaptación y crecimiento de una persona en la vida, esto es llamado flexibilidad adaptativa y aquellos que poseen un alto índice en flexibilidad adaptativa están listos y dispuestos a adaptar su estilo de aprendizaje a situación de aprendizaje.

Este atributo se refiere a la habilidad de los colaboradores de adaptarse al cambio, de tener la flexibilidad suficiente para cambiar de una dirección a otra, de una tarea a otra, de manejar cada tarea bien y además de saber priorizar (Antelo, Prilipko y Sheridan-Pereira, 2010).

\subsection{Motivación hacia el logro de resultados}

De acuerdo con Antelo, Prilipko y Sheridan-Pereira (2010), los lideres aprecian la auto motivación en los colaboradores a diferencia de la motivación artificial guiada por recompensas monetarias o similares. Los colaboradores son individuos honestos y valientes que

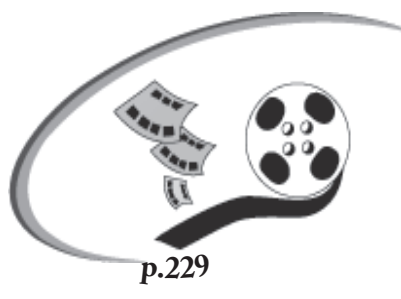
crean su propio significado de la vida en vez de perseguir las metas impuestas por la sociedad como el dinero, el poder y la fama, ellos no compiten por poder o liderazgo, ellos desean cooperar para alcanzar las metas (Litwin y Stringer, 1968; Kelley, 1992).

\section{CONCLUSIONES}

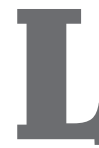
e menciona que las características de los expertos son adquiridas a través de la experiencia (Day, Harrison y Halpin, 2009) y que los colaboradores aprenden de la experiencia
(Zaleznik, 1965). Los efectos de la práctica son críticos (Chi, Glaser y Farr, 1988) tanto que Ericson (1996) y Ericson y Lehman (19986) han calculado un mínimo de horas para adquirir características a niveles de experto. 


\section{REFERENCIAS BIBLIOGRÁFICAS}

Antelo, A., Prilipko, E. V. y Henderson, R. (2011) Rainbow of Followers' Attributes in a Leadership Process. International Journal of Management and Information Systems. 15:2.

Antelo, A., Prilipko, E.V., y Sheridan-Pereira, M. (2010). Assessing effective attributes of followers in a leadership process. Paper presented at the 2010 European College Teaching and Learning Conference. Dublin, Ireland.

Aslan, S. y Erkus, A. (2008). Measurement of Emotional Intelligence: Validity and Reliability Studies of Two Scales. World Applied Science Journal. 4 (3): 430-438.

Awamleh, R. y Gardner, W.L. (1999) Perceptions of Leader Charisma and Effectiveness: The effects of vision content, delivery, and organizational performance. Leadership Quarterly, 10, 345-373.

Bennis, W. (2008) The Art of Followership. Executive Excellence, 25, 4, 4.

Brown, A. (2003) The New Followership: A Challenge for Leaders. The Futurist, 37, 2, 68.

Brown, A. y Thornborrow, W.T. (1996) Do Organizations get the Followers They Deserve? Leadership \& Organization Development Journal, 17, 1, 5-11.

Buhler, P. (1993) The Flipside of Leadership - Cultivating Followers, Supervision, 54, 17-19.

Chi, M.T.H., Glaser, R. y Farr, M.J. (1988). The nature of expertise. Hillsdale, NJ: Erlbaum.

Choi, Y. y Mai-Dalton, R. R. (1999) The Model of Followers' Responses to Self-sacrificial Leadership: An empirical test. Leadership Quarterly, 10, 397-421.

Day, D.V., Harrison, M.M., y Halpin, S.M. (2009). An integrative approach to leader development: Connecting adult development, identity, and expertise. New York, NY: Psychology Press.

Ericsson, K.A. (1996). The acquisition of expert performance: An introduction to some of the issues. In "The Road to Excellence: The Acquisition of Expert Performance in the Arts and Sciences, Sports, and Games", Ericsson, K.A., ed. Mahwah, NJ: Erlbaum.

Ericsson, K. A., y Lehmann, A.C. (1996). Expert and exceptional performance: Evidence on maximal adaptations on task constraints. Annual Review of Psychology, 47, 273-305. 
Calder, B, J. (1977) An attribution theory of leadership. In B. Staw \& G. Salancik (Eds.), New Direction in Organizational Behavior. Chicago: St. Clair Press. 179-204.

Chaleff, I. (1995) The corageous follower: Standing up to and for our leaders. San Francisco: Berrett- Koehler.

Chaleff, I. (1996). Effective Followership. Executive Excellence, 13, 4, 16-17.

Chaleff, I. (1997). Learn the Art of Followership. Executive Excellence, 29, 2, 51.

Chaleff, I. (2010). Closed Hierarchy: It often leads to catastrophe. Executive Excellence, 27, 4, 16-17.

Clydesdale, G. (2009). Management education's blind spot: Management of workplace relations. Journal of European Industrial Training, 33(2), 178-191.

Diccionario de la Lengua Española (2010) Real Academia Española. $22^{\circ}$ Edición. Consulta electrónica 4/7/2012, http://lema.rae.es/drae/?val=atributo.

Ferris, G.R., et al (2005). Development and validation of the Political Skill Inventory. Journal of Management, 31, 126-152.

Goleman, D. (1995) Inteligencia Emocional. Vergara Editor, S.A. Buenos Aires, Argentina, 1995.

Goleman, D. (2000) Leadership That Gets Results. Harvard Business Review. Mach-April. 78-90.

Grant, J. (1988). Women as managers: What they can offer to organizations. Organizational Dynamics, 56-63.

Harris, A. (2009). Creative leadership: Developing future leaders. British Educational Leadership, Management \& Administration Society (BELMAS), 23(1), 9-11.

Helgeson, S. (1990). The female advantage: Women's ways of leadership. New York: Doubleday/Currency.

Hollander, E.P., y Kelly, D.R. (1992). Appraising relational qualities of leadership and followership. International Journal of Psychology, 27, 289-290.

Hunsaker, P.L. y Cook, C. W. (1986) Managing organizational behavior. Reading, Mass: Addison - Wesley.

Katz, D. y Kahn, R.L. (1966). The social psychology of organizations. New York: John Wiley \& Sons.

Kellerman, B. (2007) What Every Leader Needs to Know about Followers. Harvard Business Review, December 2007, 84-91.

Kellerman, B. (2008) Leaders and Followers. Executive Excellence, 25, 5, 4. 
Kelley, H.H. (1973) The processes of causal attribution. American Psychologist, 28 (2), 107-128.

Kelley, R.E. (1992) The power of followership: How to create leaders people want to follow and followers who lead themselves. New York: Doubleday Currency.

Kelley, R. (2008) The Art of Followership. San Francisco, CA:USA, Wiley.

Kolb, D.A. (1984). Experiential learning: Experience as the source of learning and development. Englewood Cliffs, NJ: Prentice-Hall.

Lewin, K. (1968) Resolving social conflicts. New York,Harper.

Likert, R. (1968) El factor humano en la empresa, su dirección y valoración. Bilbao: Deusto.

Litwin, G.H. y Stringer, R. A. (1968) Motivation and organizational climate. Boston: Division of Research, Graduate School of Business Administration, Harvard University.

Loden, M. (1985) Feminine leadership: or how to succeed in business without being one of the boys. New York, NY: Times Books.

Lord, R.G. y Maher, K.J. (1991) Leadership and Information Processing: Linking perceptions and performance. Boston: Unwin-Hyman.

Lord, R.G. y Smith, J.E. (1983) Theoretical, Information Processing and Situational Factor Affecting Attribution Theory Model of Organizational Behavior. The Academy of Management Review. 8 (1). 50-60.

Lundin, S.C. y Lancaster, L.C. (1990) Beyond leadership: The importance of followership. Futurist, 24, 18-22.

Marín, M. y Troyano, Y. (2006) Trabajo con grupos. Técnicas de Intervención. Pirámide. $2^{\circ}$ Edición. España.

Mayer, J.D., y Salovey, P. (1997). What is emotional intelligence? In P. Salovey \& D.J. Sluyter (Eds.), Emotional development and emotional intelligence: Educational implications (pp.3-34). New York: Basic Books.

Meindl, J.R., et al (1985) The Romance of Leadership. Administrative Science Quarterly, 30, 78-102

Meindl, J.R. (1995) The Romance of Leadership as a Follower-Centric Theory: A Social Constructionist Approach. The Leadership Quarterly, 6 (3), 329-341.

Menkes, J. (2005). Executive intelligence: What all great leaders have. New York, NY: HarperCollins.Nunnally, J. C. (1978) Psychometric theory. New York, NY, USA: McGraw Hill.

Pacheco, R. (2005) Competencias claves para la comunicación organizacional. Revista Latinoamérica de Comunicación CHASQUI. 090, junio, 70-75. 
Peterson, S.J. y Luthans, F. (2003) The positive impact and development of hopeful leaders, Leadership and Organization Development Journal. 24:1, $26-31$.

Salovey, P. y Mayer, J.D. (1990) Emotional intelligence. Imagination, Cognition, and Personality, 9, 185-211.

Salovey, P. y Sluyter, D.J. (1997) Emotional development and emotional intelligence: Educational implications. Nueva York: Basic Books.

Senge, P. (1993) La quinta disciplina. Barcelona, Gránica,

Stogdill, R.M. (1963) Manual for the Leader Behavior Description Questionnaire-From XII. Columbus: Ohio State University, Boreau of Business Research.

Stogdill, R.M. (1974) Hansbook of Leadership. New York: Free Press.

Thornborrow, W.T. (1994) Follow my Leader: an investigation into the perceptions of follower types in the Hallifax Building Society, E.M.E. plc \& Thornstons plc, Tesis de MBA, School of Management and Finance, University of Nottingham.

Vecchio, R.P. (1987). Effective followership: Leadership turned upside down. Journal of Business Strategies, 4, 39-47.

Waldman, D.A., et al (2001) Does Leadership Matter? CEO Leadership Attributes and Profitability under conditions of Perceived Environmental Uncertainty. The Academy of Management Journal. 44 (1). 134-143.

Whetton, D.A. y Cameron, K.S. (1991) Developing management skills. New York: Harper-Collins.

Yukl, G. (2002). Leadership in organizations (5th Ed.) Upper Saddle River, NJ, USA: Prentice Hall.

Zaleznik, A. (1965) The Dynamics of Subordinacy. Harvard Business Review, May-Jun 1965, 119-131. 